Check for updates

Cite this: Mater. Adv., 2022, 3,562

Received 25th July 2021,

Accepted 11th November 2021

DOI: $10.1039 / \mathrm{d} 1 \mathrm{ma00641j}$

rsc.li/materials-advances

\title{
A polythiophene-SWCNTs self-assembled nanorobot to clean up gas molecules $\dagger$
}

\author{
Chun Li $\left(\mathbb{D}\right.$ and Kedong $\mathrm{Bi}^{*}$
}

\begin{abstract}
The storage and release of hydrogen has become the most crucial factor relating to the practical utilization of hydrogen energy, which is an extremely promising alternative energy carrier for future use. In this study, we employ steered molecular dynamics (SMD) to investigate the adhesion competition between polythiophene (PT) and hydrogen on single-walled carbon nanotubes (SWCNTs) in depth. Utilizing this adhesion competition, PT chains assembled with SWCNTs can be regarded as a nanorobot to remove gas molecules attached to the surfaces of the tubes. The release ratio of $\mathrm{H}_{2}$ could be improved to approximately $100 \%$ under high pressure when PT chains were introduced into the system to occupy the entire van der Waals (VdW) interaction space of the tubes. Remarkably, adhesion interactions between SWCNTs with different diameters and PT can be further utilized to guide the movement of nanorobots on SWCNT surfaces, consequently recovering the system storage capacity. These simulation results are highly expected to shed new light on the application of nanorobots.
\end{abstract}

\section{Introduction}

Hydrogen energy is regarded as the most promising alternative energy source to relieve energy crises and environmental pollution, which are two large stumbling blocks preventing social development, ${ }^{1,2}$ because it can be obtained at low cost and can produce high energy upon combustion with no air pollution. ${ }^{3-8}$ However, the storage and transport of hydrogen are the main barriers when it comes to practical utilization. ${ }^{9,10}$ In 1997, Dillon et al. first accomplished a milestone work in which SWCNTs were used to condense high-density hydrogen, and it was confirmed that $\mathrm{H}_{2}$ was adsorbed physically on the SWCNTs. ${ }^{11}$ After that, the storage of hydrogen on CNT-based materials has been investigated through extensive experimental and theoretical studies. ${ }^{12-17}$ As a lot of scientific effort has been made in this direction, hydrogen storage based on tubular porous materials at room temperature has continuously improved in recent years. However, these enhancements of hydrogen storage capacities are achieved through increasing the binding energy between hydrogen and the storage system, ${ }^{18,19}$ which will eventually lead to further difficulties relating to hydrogen desorption. Additionally, a working environment with high pressure or low temperature will also result in difficulties when desorbing $\mathrm{H}_{2}$ from SWCNT storage

Jiangsu Key Laboratory for Design and Manufacture of Micro-Nano Biomedical Instruments, School of Mechanical Engineering, Southeast University, Nanjing, 211189, China. E-mail: kedongbi@seu.edu.cn; Fax: +86-25-52090504; Tel: +86-25-52090501 ext. 8410

$\dagger$ Electronic supplementary information (ESI) available. See DOI: 10.1039/ d1ma00641j systems. Common methods for releasing all hydrogen molecules involve heating and depressurizing storage systems, and, to our knowledge, there have been almost no investigations into the release of $\mathrm{H}_{2}$ without changing the system temperature or pressure.

Polythiophene is a conjugated polymer with excellent mechanical properties due to its combination of backbone stiffness and aromaticity, ${ }^{20,21}$ and it has been preliminarily demonstrated to exhibit strong adhesion to various materials. ${ }^{22-26}$ Therefore, it is usually considered an effective adhesive. Interestingly, when PT encounters tubular porous materials, such as SWCNTs and boron nitride nanotubes, adhesion competition will arise between PT and any other adsorbing material, providing novel inspiration for the release of gas molecules and recovering the storage capacities of tubular porous materials.

In this work, this adhesion competition, which might also apply to other gas molecules, such as $\mathrm{N}_{2}$ and $\mathrm{CO}_{2}$, adsorbed on SWCNT-based materials, is illustrated and applied to $\mathrm{H}_{2}$ release from SWCNTs and the recovery of the $\mathrm{H}_{2}$ storage capacity of a SWCNT system to investigate the underlying mechanism. Deep insight into the details of the adhesion of both PT and hydrogen to SWCNTs is gained based on steered molecular dynamics (SMD) simulations. Based on adhesion competition between PT and hydrogen on SWCNTs, PT chains assembled with SWCNTs could be regarded as a nanorobot to release hydrogen from a CNT storage system under high pressure. Moreover, the storage ability of the system can be recovered upon further utilizing adhesion interactions between PT and SWCNTs with different diameters. 


\section{Computational methods}

All of the initial models were built using the software package Materials Studio, and the all-atom model ${ }^{27}$ was applied in the simulations, which allowed each atom to possess a particular site and mass. SWCNT models, namely $(10,10)$ and $(15,15)$, were adopted in our simulation, with diameters of around $1.36 \mathrm{~nm}$ and $2.34 \mathrm{~nm}$, respectively. The $(10,10)$ carbon nanotube consisted of 20 unit-cells while the other had 50 unit-cells. The former SWCNTs were used to store hydrogen, for the reason that the highest physisorption energy would be obtained using SWCNTs with a diameter range of 10-20 $\AA^{16,22}$ while the latter was used to remove the PT chains. The polymerization degree of the PT chains is 15 in this work. It is worth noting that the length of the PT chains was comparable to that of $(10,10)$ SWCNTs, which is beneficial for hydrogen release (Fig. S1 and S2, ESI $\dagger$ ). Both the SWCNTs and PT chains were built along the $Z$ direction, as shown in Fig. 1a and b. The simulation boxes were cuboid with $1000 \mathrm{H}_{2}$ molecules inside. To evaluate the hydrogen storage capacity under a pressure of $10 \mathrm{MPa}$ at various temperatures, a series of box sizes was built based on the ideal gas law, $p V=N k_{\mathrm{B}} T$, where $p$ is the gas pressure, $V$ is the system volume, $N$ is the number of gas molecules, $k_{\mathrm{B}}$ is the Boltzmann constant, and $T$ is the gas temperature.

For SWCNTs, PT, and $\mathrm{H}_{2}$, the atom types were adopted using the PCFF force field, ${ }^{28}$ and the force cut-off distance was $1.2 \mathrm{~nm}$. All MD simulations were performed with the LAMMPS (Large Scale Atomic/Molecular Massively Parallel Simulation) software package. $^{29}$ Periodic boundary conditions were applied in all directions, and the time step was set as 0.5 fs. All simulations were conducted in the NVT ensemble using the Nosé-Hoover thermostat ${ }^{30,31}$ to maintain the desired temperature.

In our analysis, $\mathrm{H}_{2}$ molecules adsorbed by SWCNTs were divided into two groups. Those molecules with a distance to the axis of $(10,10)$ SWCNTs of less than $6.8 \AA$ were considered as inside the SWCNTs. For the reason that the VdW radius of carbon atoms was $1.7 \AA$ and the closest distance between hydrogen molecules and the SWCNT surface was approximately $3 \AA{ }^{32}$ the molecules that were within $4.7 \AA$ of the SWCNT surface were regarded as being adsorbed on $(10,10)$ SWCNTs. To directly count the number of hydrogen molecules, we set several regions and dynamic groups separated by a series of radii from the axis of the $(10,10)$ SWCNT. The number of hydrogen molecules in each region was counted every time step, averaged over a simulation time of $0.5 \mathrm{~ns}$ when the system was in equilibrium. Each simulation was performed more than 7 times via randomly assigning different initial velocities to all atoms.

\section{Results and discussion}

\subsection{Details of PT chain and hydrogen adhesion on SWCNTs}

Deep analysis of adhesion between PT chains and SWCNTs was conducted under a hydrogen atmosphere using SMD in which conformational changes were considered. In our work, when the system reached equilibrium, the PT chains were detached from the outer and inner surfaces of both $(10,10)$ and $(15,15)$ tubes, respectively. As shown in the insets of Fig. 2a and b (Fig. S3, ESI $\dagger$ ), the PT chains were dragged at a constant moving speed away from the SWCNT surface along the axial direction of the tube by a virtual spring. During the detachment process, a reaction coordinate ${ }^{33}$ was assumed to compute the potential of mean force (PMF, $\Delta G$ ) based on Jarzynski equality. ${ }^{34}$ The detaching force was calculated based on the first derivative of $\mathrm{PMF}$, and the adhesion force (AF) was defined as the maximum detaching force.

The simulation results of PMF and the detaching force are demonstrated in Fig. 2 and Table 1. It is observed that PT chains exhibit strong adhesion to both the outer and inner surfaces of SWCNTs. For the PT- $(10,10)$ SWCNTs system, the free energy change $(\Delta G)$ values are $61.7 \mathrm{kcal} \mathrm{mol}^{-1}$ and $120 \mathrm{kcal} \mathrm{mol}^{-1}$ for the outer and inner surfaces, respectively, in the detaching (a)

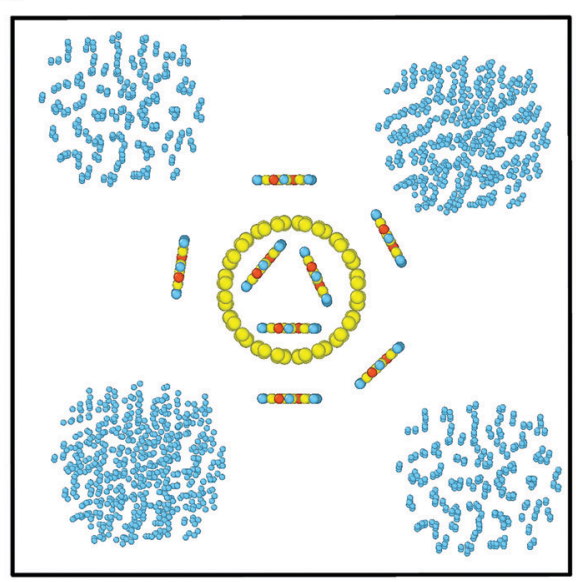

(b)

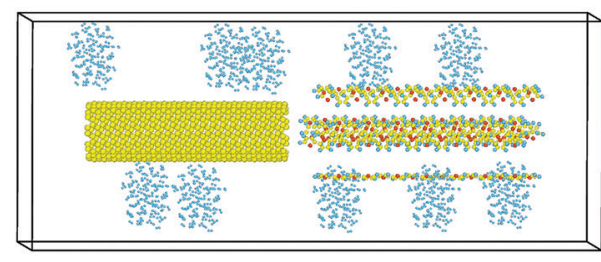

(c)

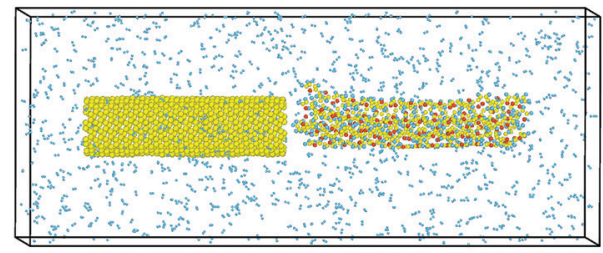

Fig. 1 The initial model consisting of hydrogen, $(10,10)$ SWCNTs, and PT chains. (a) A top view and (b) side view. (c) A snapshot of the simulation box (side view) after a simulation time of $0.5 \mathrm{~ns}$. Blue, yellow, and red represent $\mathrm{H}, \mathrm{C}$, and $\mathrm{S}$ atoms, respectively. 
(a)

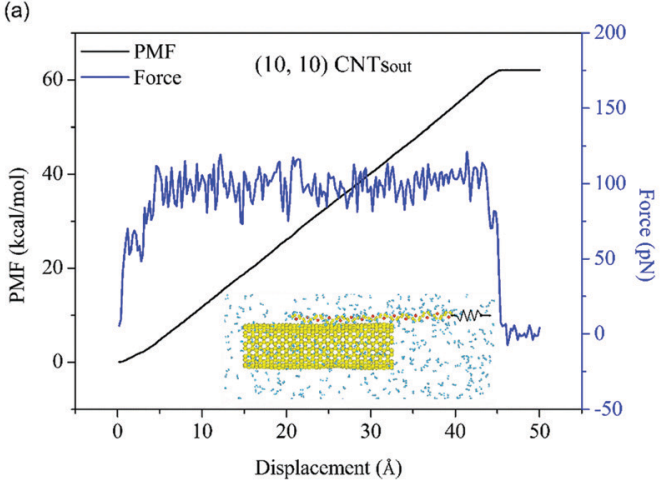

(c)

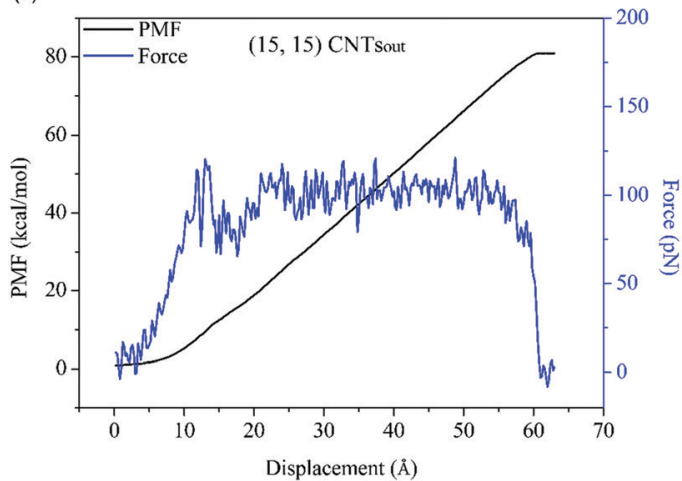

(b)

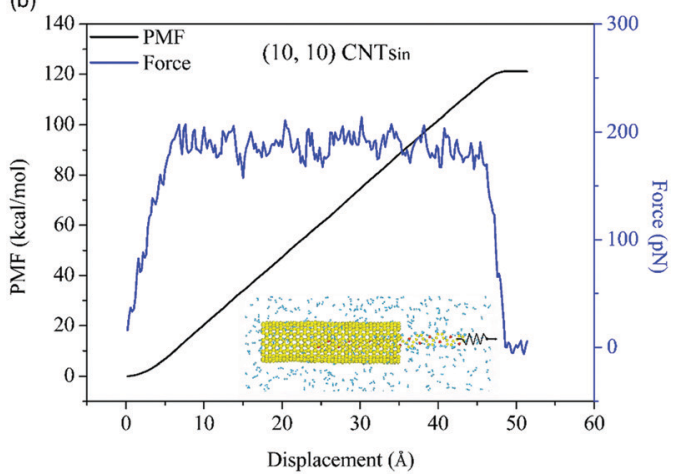

(d)

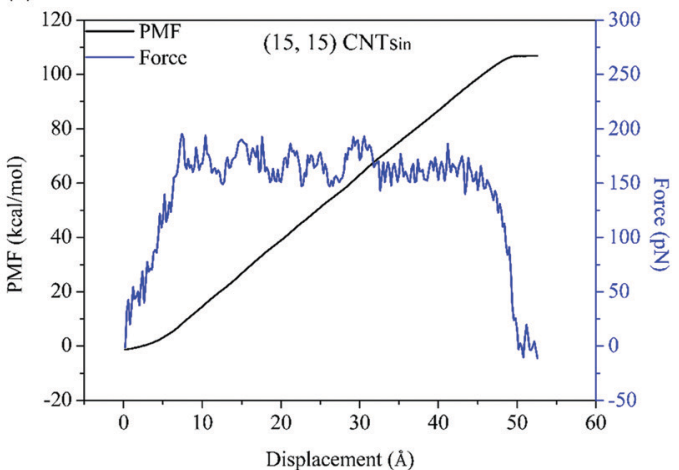

Fig. 2 The potential of mean force profiles and detaching force curves of PT-SWCNTs systems for the (a and c) outer and (b and d) inner surfaces of (a and $b)(10,10)$ and $(c$ and d) $(15,15)$ tubes.

Table 1 Energy change and adhesion force values for each system during the desorption of PT

\begin{tabular}{lcclc}
\hline $\begin{array}{l}\text { Energy } \\
\left(\mathrm{kcal} \mathrm{mol}^{-1}\right)\end{array}$ & $\begin{array}{l}\Delta G \\
(\mathrm{kcal} \mathrm{mol}\end{array}$ & $\begin{array}{l}\Delta E \\
\left(\mathrm{kcal} \mathrm{mol}^{-1}\right)\end{array}$ & $\begin{array}{l}T \Delta S \\
\left(\mathrm{kcal} \mathrm{mol}^{-1}\right)\end{array}$ & $\mathrm{AF}(\mathrm{pN})$ \\
\hline$(10,10) \mathrm{CNTs}_{\text {out }}$ & 61.7 & 85 & 23.3 & 99.4 \\
$(15,15) \mathrm{CNTs}_{\text {out }}$ & 84.4 & 99.5 & 15.1 & 108 \\
$(15,15) \mathrm{CNTs}_{\text {in }}$ & 107 & 132.3 & 25.3 & 166.9 \\
$(10,10) \mathrm{CNTs}_{\text {in }}$ & 120 & 152 & 32 & 188.5
\end{tabular}

process, which are attributed to different interfacial adhesion forces between the PT chains and the tubes. Compared to PT atoms outside the tube, more carbon atoms from $(10,10)$ SWCNTs interact with PT atoms inside the tube within the force cut-off distance, causing the larger adhesion force on the inner surface, as shown in Table 1. In addition, the corresponding interaction energy changes of the hydrogen molecules with SWCNTs are both less than $10 \mathrm{kcal} \mathrm{mol}^{-1}$ in these aforementioned adhesion processes. Furthermore, adsorption and desorption are a pair of reversible processes. Therefore, the PT chains would spontaneously wrap around and insert into the SWCNTs, driving away hydrogen due to adhesion competition at the tube surfaces. In addition, the entropy decrease during adsorption indicates that PT chains would probably be aligned in an orderly manner on the tube surface; the entropy change can be obtained from the formula:

$$
\Delta G=\Delta E-T \Delta S
$$

where $\Delta E$ is the potential energy change and $\Delta S$ is the entropy change.

The existence of two distinctive adhesion performances provides a feasible method for entirely removing $\mathrm{H}_{2}$ molecules stored both inside and outside of SWCNTs with the lowest number of PT chains. Compared with the system above, the free energy changes in the PT- $(\mathbf{1 5}, \mathbf{1 5})$ SWCNTs system are $84.4 \mathrm{kcal} \mathrm{mol}^{-1}$ and $107 \mathrm{kcal} \mathrm{mol}^{-1}$ for outer and inner surfaces, respectively. Based on the same reasoning, the tube surfaces in this system show a higher adhesion force than the outer surfaces of $(10,10)$ SWCNTs. This adhesion force difference results in adhesion competition between different SWCNTs and PT, thus providing a novel way to control the movement of PT chains on tube surfaces. Taking advantage of the adhesion competition between PT and hydrogen on SWCNTs, we conceive of a complete program, first utilizing the assembly of PT chains with SWCNTs to construct a nanorobot to remove $\mathrm{H}_{2}$ molecules from tube surfaces and then controlling the nanorobot to leave the surfaces.

\subsection{Hydrogen release using PT chains}

We first simulated the storage of hydrogen using $(10,10)$ SWCNTs at three representative temperatures, $77 \mathrm{~K}, 273.15 \mathrm{~K}$, and $300 \mathrm{~K}$, to ascertain changes in the hydrogen release behavior with temperature. For all systems, the environmental pressure was maintained at $10 \mathrm{MPa}$ through varying the box size. The model configuration was similar to the model depicted in Fig. 1, but without PT chains in the box. The $\mathrm{H}_{2}$ 
molecules were placed more than $10 \AA$ from the SWCNTs to ensure that few interactions existed between them at the beginning, and all these $\mathrm{H}_{2}$ molecules quickly diffused in the box within 1 ps. After 250 ps, the $\mathrm{H}_{2}$ molecules reached a relatively stable state and were partly bound to the inner and outer surfaces of the SWCNTs.

The final snapshots of the adsorption states at different temperatures are shown in Fig. 3a-c. It is easily observed that the number of adsorbed $\mathrm{H}_{2}$ molecules decreases with an increase in temperature. The total number of adsorbed hydrogen molecules distributed along the SWCNT radius is depicted in Fig. 3d. A radius distance of $11.5 \AA$ to the axis of the SWCNTs is selected as the critical size for hydrogen adsorption on SWCNTs. The weight percentages of adsorbed $\mathrm{H}_{2}$ molecules based on the total mass are calculated to be $6.50 \%, 1.20 \%$, and $1.02 \%$ at $77 \mathrm{~K}$, $273.15 \mathrm{~K}$, and $300 \mathrm{~K}$, respectively, in this work, which are comparable to the existing literature. ${ }^{15,35,36}$ In other words, the release ratio is $84.31 \%$ when the temperature increases from $77 \mathrm{~K}$ to $300 \mathrm{~K}$ at a constant pressure of $10 \mathrm{MPa}$. It is noteworthy that there is still around $15.69 \%$ residual hydrogen adsorbed on SWCNTs at $300 \mathrm{~K}$, as exhibited in Fig. 3c. This residual gas results in wasted storage space, and the complete release of hydrogen requires a further increased temperature or reduced pressure, which is inconvenient for the application of hydrogen storage using CNT-based systems in strict working environments.

Subsequently, PT chains were introduced into the SWCNT system above to further release hydrogen at $300 \mathrm{~K}$ and $10 \mathrm{MPa}$. As illustrated in Fig. 1a and b, the new system consisted of $1000 \mathrm{H}_{2}$ molecules scattered in several areas, $(10,10)$ SWCNTs, and a set of PT chains that were distributed along the tube axis.

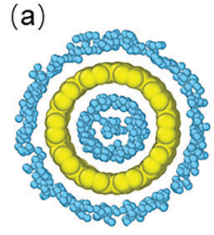

$77 \mathrm{~K}$ (b)

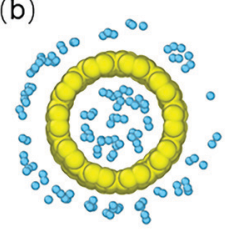

$273.15 K$

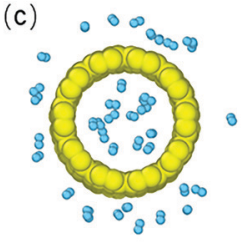

$300 \mathrm{~K}$ (d)

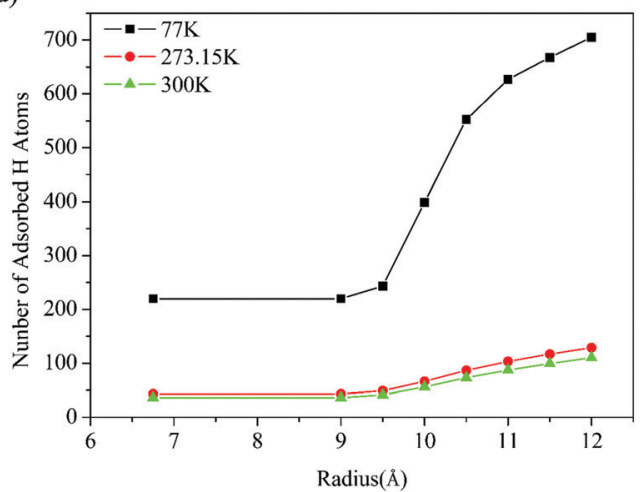

Fig. 3 (a-c) Final snapshots (top view) of the adsorption states of hydrogen at different temperatures. (d) The numbers of hydrogen molecules adsorbed by $(10,10)$ SWCNTs under $10 \mathrm{MPa}$ at different temperatures.
The minimum distance between a single chain and its neighbor chains was within the VdW cut-off radius and the closest distance from the carbon atoms of SWCNTs to the sulfur atoms of PT chains was about $5 \AA$ A. These configurations were constructed for the reason that pre-existing interactions between them gave rise to the introduction of PT chains into the tube quickly. Simulations were performed on a series of storage systems with different numbers of PT chains, and six representative samples, with the introduction of $1,2,3,4,8$, and 12 chains, respectively, were analyzed to gain more insight into the hydrogen release performance.

During the initial pre-equilibration time of $2 \mathrm{~ns}$, PT chains were fixed to allow the hydrogen molecules to fully diffuse over the entire simulation box at $300 \mathrm{~K}$ and $10 \mathrm{MPa}$. Hydrogen molecules were counted when $\mathrm{H}_{2}$ adsorption reached saturation on $(10,10)$ SWCNTs, and the number of adsorbed molecules was comparable to that in the aforementioned initial system without PT chains. Subsequently, the PT chains were freed to generate a new equilibrium scenario in the following $2.5 \mathrm{~ns}$. As manifested in Fig. 1c, PT chains converged into a bundle at the beginning before entering the tube owing to the stronger attraction between these chains compared to between the chains and SWCNTs. Thereafter, all of the PT chains gradually approached the end-face of the tube and started to increasingly interact with the SWCNTs. It is noticed that PT chains spontaneously move into the tube, mainly along the axial direction of the SWCNTs, due to their strong adhesion force with the tube.

The residual hydrogen configurations for these 6 samples are illustrated in Fig. 4. As expected, the PT chains are indeed aligned in order on the tube surfaces. It is also found that the PT chains prefer to enter the tube, as the adhesion of PT chains to the inside of the tube is stronger than to the outside, as presented in Table 1, which can also be easily predicted based on the binding energy between them (Fig. 5b). In addition, only 3 PT chains at most are able to enter inside the tube, while the others wrap around the tube because of the limited inner space in $(10,10)$ SWCNTs, as demonstrated in Fig. $4 d-f$. In both cases, PT chains occupy the positions where hydrogen molecules were located and drive away most residual $\mathrm{H}_{2}$ (a)

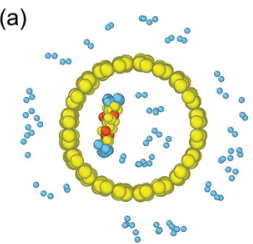

(d)

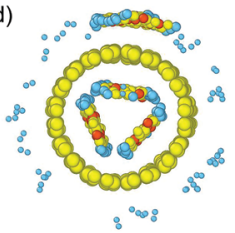

(b)

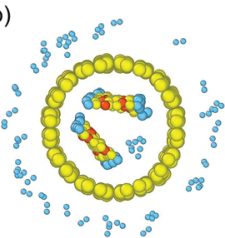

(e)

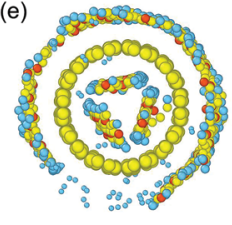

(c)

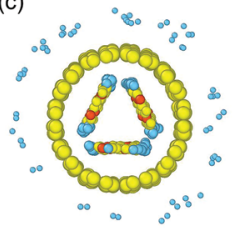

(f)

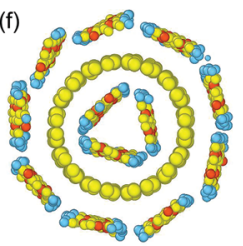

Fig. 4 Snapshots (end-face of the tube) of residual hydrogen on SWCNTs with an increasing number of chains: (a) 1, (b) 2, (c) 3, (d) 4, (e) 8, and (f) 12 PT chains. 
(a)

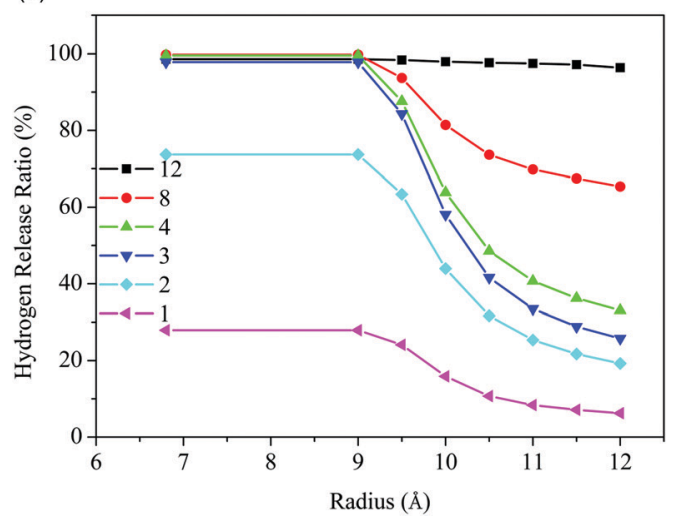

(b)

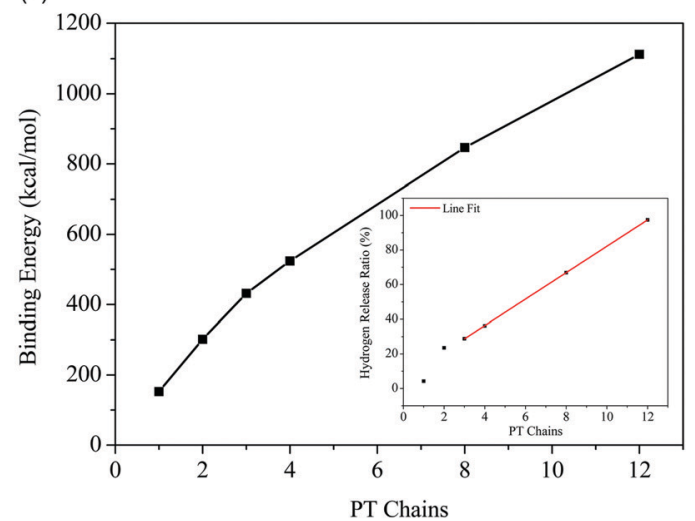

Fig. 5 (a) Hydrogen release ratios along the radial direction of SWCNTs with different numbers of chains. (b) The hydrogen release ratios $11.5 \AA$ from the tube axis (inset) and the binding energy dependence on the number of PT chains.

adsorbed on the tube as a result of adhesion competition. The hydrogen release ratios along the radial direction of the SWCNTs for these six systems are summarized in Fig. 5a. It is observed that the release ratios present a downward trend along the radial direction of the tube for all the systems.
Additionally, the hydrogen release performance is enhanced as the number of chains increases, because more and more $\mathrm{H}_{2}$ space on tube surfaces is occupied by polymer chains after adhesion competition, as shown in Fig. 4, which is confirmed based on the binding energy increase shown in Fig. 5b.

(a)
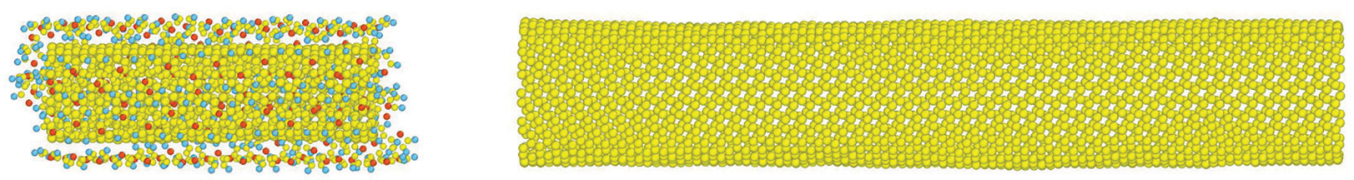

(b)

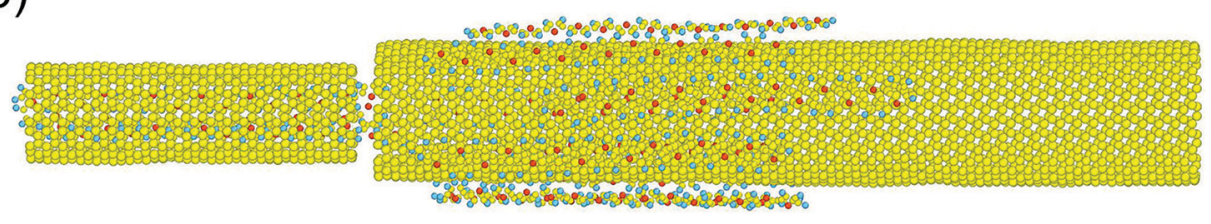

(c)

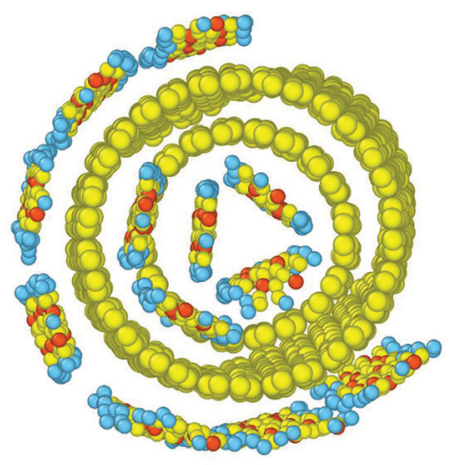

(d)

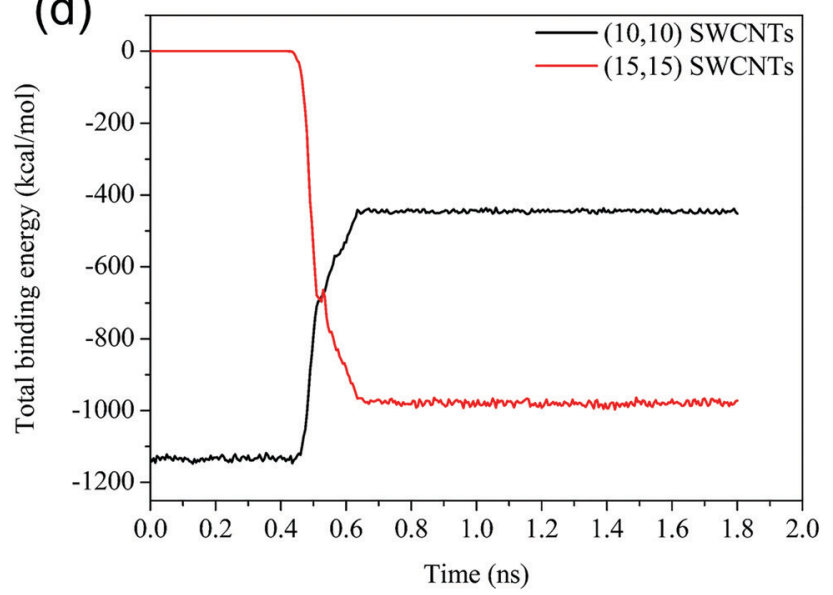

Fig. 6 (a) The initial configuration (side view) with 12 PT chains attached to a $(10,10)$ SWCNT. The final configuration of the system: (b) side view and (c) top view (end-face of the tube). (d) Changes in the total binding energies between the PT chains and SWCNTs. 
This enhancement converts from nonlinear to approximately linear when the number of chains is more than three. Eventually, the hydrogen release ratio is close to $100 \%$ when 12 PT chains are introduced into the system (inset of Fig. 5b), indicating that 12 PT chains are sufficient to occupy the entire VdW interaction space of $(10,10)$ SWCNTs, as demonstrated in Fig. 4f.

For SWCNTs in the diameter range of $10-20 \AA$, due to the orderly arrangement of PT chains on the tube surface, the number of PT chains that would be enough to occupy the entire VdW interaction space of the tube can be derived (details in the ESI $\dagger$ ) as follows:

$$
\begin{aligned}
N_{\text {out }} & =\frac{90}{\arcsin \left(\frac{3.4}{d+6.8}\right)} \\
N_{\text {in }} & =\frac{90}{\arcsin \left(\frac{3.4}{d-6.8}\right)}
\end{aligned}
$$

where $N_{\text {out }}$ and $N_{\text {in }}$ are the numbers of PT chains outside and inside the tube, respectively, and $d$ is the diameter of the SWCNTs. For the case of $(10,10)$ SWCNTs, our calculations predict that $N_{\text {out }}$ is 9.38 and $N_{\text {in }}$ is 3, consistent with the simulation results in this work.

\subsection{Removing PT chains via adhesion competition}

To recover the hydrogen storage capacity of $(10,10)$ SWCNTs, it was necessary to remove the PT chains from the tube. Considering that the evolution of the system is towards a reduction in free energy to achieve a more stable state, we introduced $(15,15)$ SWCNTs into the above system to remove the chains from the $(10,10)$ SWCNT surface. For simplification, hydrogen was assumed to be completely desorbed from the $(10,10)$ SWCNTs system. As depicted in Fig. 6a, the distance between the adjacent ends of the two tubes was set to about $20 \AA$ in the initial stage. As $(15,15)$ SWCNTs approached $(10,10)$ SWCNTs gradually, the large-diameter tube interacted with the PT chains first because the PT chains were a little longer than $(10,10)$ SWCNTs. When the aforementioned distance was reduced to around $5 \AA$, both SWCNTs were fixed at the same time. Subsequently, the PT chains completed their transfer spontaneously within the next $150 \mathrm{ps}$.

As illustrated in Fig. 6c, when the new system reaches equilibrium, seven chains adhere in an orderly manner to the outside of $(15,15)$ SWCNTs, and two chains enter inside this tube. All of the PT chains adsorbed on the outer surface of $(10,10)$ SWCNTs transfer spontaneously to both the inner and outer surfaces of $(15,15)$ SWCNTs due to adhesion competition. This situation is because the interfacial adhesion forces of PT chains on the outside and inside of $(15,15)$ SWCNTs are $108 \mathrm{pN}$ and $166.9 \mathrm{pN}$, respectively, higher than on the outside surface of $(10,10)$ SWCNTs $(99.4 \mathrm{pN})$, as shown in Table 1 . Driven by the stronger adhesion, the system achieves a more stable state in which the total binding energy between 12 PT chains and SWCNTs increases by about $300 \mathrm{kcal} \mathrm{mol}^{-1}$ from the initial state, as demonstrated in Fig. 6d. Furthermore, the AF between the PT chains and the inner surface of the $(10,10)$ tube, which is higher than that between the PT chains and $(15,15)$ SWCNTs, keeps three PT chains from transferring to the thicker tube, as expected. In the same way, these three chains could be removed by a smaller tube that exhibits stronger AF with the PT chains due to the deeper VdW potential well ${ }^{37}$ inside the tube caused by forcefield overlap.

\section{Conclusions}

In summary, a detailed investigation into adhesion between PT chains and SWCNTs under a hydrogen atmosphere is carried out based on SMD simulations. Based on adhesion competition, a novel method is proposed, introducing PT chains into a SWCNTbased hydrogen storage system to construct a nanorobot which can remarkably improve the hydrogen release capacity. The release ratio of hydrogen is improved to approximately $100 \%$ at 10 MPa when 12 PT chains are introduced to $(10,10)$ SWCNTs. In particular, taking advantage of the adhesion interactions involving SWCNTs with different diameters, these PT chains can be spontaneously removed to recover the system storage capacity. The inspiring simulation results presented in this work are expected to improve hydrogen energy utilization from SWCNTbased storage systems.

\section{Conflicts of interest}

The authors declare no competing financial interests.

\section{Acknowledgements}

The authors are grateful for financial support from the National Natural Science Foundation of China (51975120) and the Program to Cultivate Middle-aged and Young Science Leaders of Colleges and Universities of Jiangsu Province, China. We thank Mengtao Gu from the School of Energy and Environment of Southeast University for support with Materials Studio.

\section{References}

1 S. M. Shafie, T. M. I. Mahlia, H. H. Masjuki and A. Andriyana, Current energy usage and sustainable energy in Malaysia: A review, Renewable Sustainable Energy Rev., 2011, 15(9), 4370-4377.

2 T. N. Veziroglu and S. Sahin, 21st Century's energy: Hydrogen energy system, Energy Convers. Manage., 2008, 49(7), 1820-1831.

3 M. Z. Jacobson, W. G. Colella and D. M. Golden, Cleaning the air and improving health with hydrogen fuel-cell vehicles, Science, 2005, 308(5730), 1901-1905.

4 L. Schlapbach and A. Zuttel, Hydrogen-storage materials for mobile applications, Nature, 2001, 414(6861), 353-358. 
5 E. Tylianakis, E. Klontzas and G. E. Froudakis, Multi-scale theoretical investigation of hydrogen storage in covalent organic frameworks, Nanoscale, 2011, 3(3), 856-869.

6 Y. Luo, K. Ren, S. K. Wang, J. P. Chou, J. Yu, Z. M. Sun and M. L. Sun, First-Principles Study on Transition-Metal Dichalcogenide/BSe van der Waals Heterostructures: A Promising Water-Splitting Photocatalyst, J. Phys. Chem. C, 2019, 123(37), 22742-22751.

7 P. Ganguly, M. Harb, Z. Cao, L. Cavallo, A. Breen, S. Dervin, D. D. Dionysiou and S. C. Pillai, 2D Nanomaterials for Photocatalytic Hydrogen Production, ACS Energy Lett., 2019, 4(7), 1687-1709.

8 H. X. Wang, W. W. Fu, X. H. Yang, Z. Y. Huang, J. Li, H. J. Zhang and Y. Wang, Recent advancements in heterostructured interface engineering for hydrogen evolution reaction electrocatalysis, J. Mater. Chem. A, 2020, 8(15), 6926-6956.

9 S. Hynek, W. Fuller and J. Bentley, Hydrogen storage by carbon sorption, Int. J. Hydrogen Energy, 1997, 22(6), 601-610.

10 R. Strobel, J. Garche, P. T. Moseley, L. Jorissen and G. Wolf, Hydrogen storage by carbon materials, J. Power Sources, 2006, 159(2), 781-801.

11 A. C. Dillon, K. M. Jones, T. A. Bekkedahl, C. H. Kiang, D. S. Bethune and M. J. Heben, Storage of hydrogen in single-walled carbon nanotubes, Nature, 1997, 386(6623), 377-379.

12 P. Chen, X. Wu, J. Lin and K. L. Tan, High H-2 uptake by alkali-doped carbon nanotubes under ambient pressure and moderate temperatures, Science, 1999, 285(5424), 91-93.

13 C. Liu, Y. Y. Fan, M. Liu, H. T. Cong, H. M. Cheng and M. S. Dresselhaus, Hydrogen storage in single-walled carbon nanotubes at room temperature, Science, 1999, 286(5442), 1127-1129.

14 B. H. Chen, Mechanical response of hydrogen-filled singlewalled carbon nanotubes under torsion, Int. J. Hydrogen Energy, 2014, 39(3), 1382-1389.

15 D. P. Cao and W. C. Wang, Storage of hydrogen in singlewalled carbon nanotube bundles with optimized parameters: Effect of external surfaces, Int. J. Hydrogen Energy, 2007, 32(12), 1939-1942.

16 K. Wenelska, B. Michalkiewicz, X. C. Chen and E. Mijowska, Pd nanoparticles with tunable diameter deposited on carbon nanotubes with enhanced hydrogen storage capacity, Energy, 2014, 75, 549-554.

17 X. X. Wang, B. Y. Li, D. R. Bell, W. F. Li and R. H. Zhou, Hydrogen and methane storage and release by MoS2 nanotubes for energy storage, J. Mater. Chem. A, 2017, 5(44), 23020-23027.

18 A. Ferre-Vilaplana, Ab initio computational investigation of physisorption of molecular hydrogen on achiral singlewalled carbon nanotubes, J. Chem. Phys., 2005, $122(21), 214724$.

19 Y. Okamoto and Y. Miyamoto, Ab initio investigation of physisorption of molecular hydrogen on planar and curved graphenes, J. Phys. Chem. B, 2001, 105(17), 3470-3474.
20 G. Q. Shi, S. Jin, G. Xue and C. Li, A Conducting Polymer Film Stronger Than Aluminum, Science, 1995, 267(5200), 994-996.

21 S. Jin, S. X. Cong, G. Xue, H. M. Xiong, B. Mansdorf and S. Z. D. Cheng, Anisotropic polythiophene films with high conductivity and good mechanical properties via a new electrochemical synthesis, Adv. Mater., 2002, 14(20), 1492.

22 W. Z. Yuan, J. Z. Sun, Y. Q. Dong, M. Haussler, F. Yang, H. P. Xu, A. J. Qin, J. W. Y. Lam, Q. Zheng and B. Z. Tang, Wrapping carbon nanotubes in pyrene-containing poly(phenylacetylene) chains: Solubility, stability, light emission, and surface photovoltaic properties, Macromolecules, 2006, 39(23), 8011-8020.

23 T. Casagrande, P. Imin, F. Y. Cheng, G. A. Botton, I. Zhitomirsky and A. Adronov, Synthesis and Electrophoretic Deposition of Single-Walled Carbon Nanotube Complexes with a Conjugated Polyelectrolyte, Chem. Mater., 2010, 22(9), 2741-2749.

24 S. S. Tallury and M. A. Pasquinelli, Molecular Dynamics Simulations of Polymers with Stiff Backbones Interacting with Single-Walled Carbon Nanotubes, J. Phys. Chem. B, 2010, 114(29), 9349-9355.

25 M. Foroutan and A. T. Nasrabadi, Investigation of the Interfacial Binding between Single-Walled Carbon Nanotubes and Heterocyclic Conjugated Polymers, J. Phys. Chem. $B, 2010,114(16), 5320-5326$.

26 Y. C. Qian, M. Guo, C. Li, K. D. Bi and Y. F. Chen, New Insight on the Interface between Polythiophene and Semiconductors via Molecular Dynamics Simulations, ACS Appl. Mater. Interfaces, 2019, 11(33), 30470-30476.

27 A. S. Henry and G. Chen, Spectral phonon transport properties of silicon based on molecular dynamics Simulations and lattice dynamics, J. Comput. Theor. Nanosci., 2008, 5(2), 141-152.

28 H. Sun, S. J. Mumby, J. R. Maple and A. T. Hagler, An AbInitio Cff93 All-Atom Force-Field For Polycarbonates, J. Am. Chem. Soc., 1994, 116(7), 2978-2987.

29 S. Plimpton, Fast Parallel Algorithms for Short-Range Molecular Dynamics, J. Comput. Phys., 1995, 117(1), 1-19.

30 S. Nose, A Molecular-Dynamics Method for Simulations in the Canonical Ensemble, Mol. Phys., 1984, 52(2), 255-268.

31 W. G. Hoover, Canonical Dynamics - Equilibrium PhaseSpace Distributions, Phys. Rev. A: At., Mol., Opt. Phys., 1985, 31(3), 1695-1697.

32 Z. Ozturk, C. Baykasoglu, A. T. Celebi, M. Kirca, A. Mugan and A. C. To, Hydrogen storage in heat welded random CNT network structures, Int. J. Hydrogen Energy, 2015, 40(1), 403-411.

33 S. Park and K. Schulten, Calculating potentials of mean force from steered molecular dynamics simulations, J. Chem. Phys., 2004, 120(13), 5946-5961.

34 C. Jarzynski, Nonequilibrium equality for free energy differences, Phys. Rev. Lett., 1997, 78(14), 2690-2693.

35 A. Zuttel, P. Sudan, P. Mauron, T. Kiyobayashi, C. Emmenegger and L. Schlapbach, Hydrogen storage in 
carbon nanostructures, Int. J. Hydrogen Energy, 2002, 27(2), 203-212.

36 Y. Ye, C. C. Ahn, C. Witham, B. Fultz, J. Liu, A. G. Rinzler, D. Colbert, K. A. Smith and R. E. Smalley, Hydrogen adsorption and cohesive energy of single-walled carbon24020.nanotubes, Appl. Phys. Lett., 1999, 74(16), 2307-2309.

37 H. J. Gao, Y. Kong, D. X. Cui and C. S. Ozkan, Spontaneous insertion of DNA oligonucleotides into carbon nanotubes, Nano Lett., 2003, 3(4), 471-473. 